\title{
Is faster always better? What is the implication of a shorter time to imaging with tetrofosmin compared to sestamibi?
}

\author{
Kelly Laipply, MD, ${ }^{\mathrm{a}}$ and Myron Gerson, $\mathrm{MD}^{\mathrm{a}}$ \\ a Division of Cardiovascular Health and Disease, University of Cincinnati Medical Center, \\ Cincinnati, $\mathrm{OH}$
}

Received Apr 9, 2020; accepted Apr 9, 2020

doi: $10.1007 / \mathrm{s} 12350-020-02143-y$

\section{See related article, https://doi.org/10.10 07/s12350-020-02093-5.}

Myocardial perfusion imaging (MPI) using single photon emission computed tomography (SPECT) was revolutionized by the development and commercial availability of technetium-99m (Tc-99m) labeled radiotracers. For many decades, thallium-201 was the predominant radiotracer utilized for SPECT MPI which, compared to other radiotracers of the time, offered favorable physiologic and biologic characteristics including robust myocardial uptake, rapid blood pool clearance, near-linear relationship between uptake and regional myocardial blood flow for flows less than $2.5 \mathrm{~mL} \cdot \mathrm{min}^{-1} \cdot \mathrm{g}^{-1}$, and myocardial washout and redistribution properties which aided in distinguishing areas of non-viability from ischemic myocardium. However, disadvantages and physical limitations of thallium-201 including a predominant daughter emission $(95 \%)$ of low energy, easily scattered characteristic x-rays (69 to $81 \mathrm{keV}$ ) and a 73.1 hour physical half-life fueled the development of radiotracers with superior properties for gamma camera scintillation imaging and minimizing radiation dose to patients. ${ }^{1}$

Compared to thallium-201, Tc-99m labeled tracers feature a predominant gamma emission with energy centered at $140 \mathrm{keV}$, yielding comparatively less Compton scatter and a more favorable photopeak for modern scintillation cameras. Additionally, a

\footnotetext{
Funding No funding received from any organization for this paper. Reprint requests: Kelly Laipply, MD, Division of Cardiovascular Health and Disease, University of Cincinnati Medical Center, 234 Goodman Ave., Cincinnati, OH 45219; laipplkr@ucmail.uc.edu J Nucl Cardiol 2021;28:2629-32.

$1071-3581 / \$ 34.00$

Copyright (c) 2020 American Society of Nuclear Cardiology.
}

significantly shorter physical half-life of 6 hours allows for substantially higher administered activity of technetium-99m, leading to superior count statistics yet still lower total radiation dose to patients. Improved image resolution and count statistics, shorter acquisition times, greater image quality, and improved diagnostic accuracy make the overall superiority of technetium-99m labeled tracers over thallium-201 rarely contested in the modern era. As a result, technetium- $99 \mathrm{~m}$ radiotracer protocols have largely replaced thallium-201 and dual-isotope protocols in modern nuclear cardiology practice.

The two technetium SPECT tracers widely used today are Tc-99m-sestamibi and Tc-99m-tetrofosmin. While other Tc-99m labeled tracers have been utilized in the past and with others under current investigation, sestamibi and tetrofosmin have maintained sustained clinical use for decades. Sestamibi was approved for use by the US FDA in 1990 under the brand name Cardiolite ${ }^{\mathrm{TM}}$, with generic use of the radiotracer gaining FDA approval in 2008. As an isonitrile family molecule, the Tc- $99 \mathrm{~m}$ isomer is centrally bound to six methoxyisobutylisonitrile (MIBI) groups and binds electrostatically to mitochondria within cardiomyocytes. ${ }^{2}$ Sixty minutes after sestamibi injection, activity within the blood pool clears to $1.1 \pm 0.01 \%$ of activity injected at rest and $0.7 \pm 0.1 \%$ of activity injected during treadmill exercise. Myocardial activity remains relatively stable over time, with $74 \pm 12 \%$ of initial activity remaining present within the heart at 3 hours. ${ }^{3}$ With the radiotracer cleared from the body enterohepatically, the most intense activity occurs within the liver and gallbladder causing subdiaphragmatic uptake to be the most common source of artifact related to extracardiac activity. The heart-to-liver activity ratio for sestamibi is $0.83 \pm 0.16$ at 5 minutes post-injection rising to $1.08 \pm$ 0.27 at 60 minutes post-injection. ${ }^{4}$ These biodistribution characteristics contribute to the current ASNC guideline that time from dose injection to image acquisition for 
SPECT MPI using Tc-99m-sestamibi be delayed a minimum of 45 to 60 minutes for rest images, 15 to 20 minutes for exercise stress images, and 60 minutes for pharmacologic stress images. ${ }^{5}$

Tetrofosmin, a Tc-99m labeled diphosphine complex, was approved by the US FDA for myocardial perfusion imaging in 1996 under the brand name Myoview $^{\mathrm{TM}}$. There is currently no approved generic availability of this tracer. Tetrofosmin shows similar myocardial uptake and retention kinetics to sestamibi as well as similar relationship between myocardial uptake and regional myocardial blood flow. Blood pool clearance is significantly more rapid with tetrofosmin, with only $0.8 \%$ of peak injected activity remaining in the blood pool at 15 minutes post-injection. Though tetrofosmin is also enterohepatically cleared, clearance from the liver is more rapid than with sestamibi. These features combine to produce a favorable heart-toextracardiac activity ratio sooner than sestamibi. The heart-to-liver activity ratio with tetrofosmin is $1.04 \pm$ 0.24 as early as 5 minutes post-injection and $1.51 \pm 0.44$ after 60 minutes. ${ }^{4}$ More rapid blood pool and hepatic clearance allow for the possibility of a shorter delay time from radiotracer injection to image acquisition, with the current ASNC guideline recommending minimum delays of 30-45 minutes for rest, 10-15 minutes for exercise stress, and 45 minutes for pharmacologic stress, overall 15 minutes sooner than sestamibi. ${ }^{5}$

The continued widespread use of both Tc- $99 \mathrm{~m}$ labeled radiotracers for SPECT MPI underscores their many kinetic and radiometric similarities, but the difference in rates of clearance of extracardiac activity, particularly hepatic, has raised clinical questions on the comparative quality of images produced with variable post-injection delays. The enduring goal of seeking the "ideal" SPECT radiotracer has fueled a variety of studies comparing sestamibi and tetrofosmin. The scope of influence of this kind of comparative research is undeniable; SPECT MPI is the most commonly used non-invasive test for the evaluation of patients with known or suspected obstructive coronary artery disease worldwide, with millions of scans produced annually in the USA alone. ${ }^{6}$ In the USA, roughly 7 million nuclear cardiology procedures of which the majority were stress myocardial perfusion scans were performed in 1999, rising to 11 million by $2005 .^{7}$ In one study comparing population and SPECT MPI utilization, the USA performed greater than 1000 myocardial perfusion studies per 100,000 people in $2008 .{ }^{6}$ According to an International Marketing Ventures (IMV) report, about 8 million myocardial perfusion scans were performed in the USA across more than 5,000 nuclear cardiology labs in 2012.
An evidence-based signal of superiority between two otherwise very similar tracers could indeed have a large impact on practice patterns.

In this issue of the Journal of Nuclear Cardiology, Duvall and associates ${ }^{8}$ submit a systematic review of the existing literature comparing the use of sestamibi and tetrofosmin with respect to variable post-injection delays to image acquisition. They summarize the composite results based on subjective image quality, semiquantitative heart-to-extracardiac activity ratios, re-scan rates, and overall test efficiency. In particular, the review focuses on comparisons between MPI protocols using a shortened post-injection delay time of 15-30 minutes with tetrofosmin against longer 45-60 minute delays using both sestamibi and tetrofosmin. After screening for applicability and risk of bias, 17 studies totaling 4835 patients were included in the review. The studies are highly variable in size, design, and conclusions. Of the 4 studies comparing subjective image quality between earlier tetrofosmin and later sestamibi imaging, no difference was detected in 2 studies and superiority of each tracer was reported in the others. Heart-to-liver activity ratios were higher with tetrofosmin than sestamibi for all post-injection delays, reaching statistical significance after 30 minutes. In a single study by Ravizzini and associates, comparative re-scanning rates were lower with earlier tetrofosmin imaging compared to later sestamibi imaging; however, the blinded comparisons for the need for re-scanning were with different patients receiving each radiotracer. ${ }^{9}$ Duvall and associates conclude that earlier tetrofosmin imaging following radiotracer injection is non-inferior to later sestamibi imaging.

While the non-inferiority of early tetrofosmin imaging compared to later sestamibi imaging is supported by the data included in the present review, noninferiority of early tetrofosmin imaging compared to a standard longer delay with tetrofosmin is less compelling. In fact, one-third of the studies included in the review show superior image quality with a longer tetrofosmin post-injection delay, and half demonstrate higher heart-to-extracardiac activity ratios with a longer delay. Two of the three studies with tetrofosmin injection at rest demonstrated higher heart-to-liver ratios with a longer delay. The present study does not directly compare identically delayed tetrofosmin imaging against delayed sestamibi imaging, nor does it compare early tetrofosmin imaging against equally early sestamibi imaging.

In response to this review, nuclear cardiology providers and laboratories may consider transition of use of sestamibi to tetrofosmin, or shorten protocols if already 
using tetrofosmin, in order to improve scan and lab efficiency. Care must be taken, however, in interpreting the results of this systematic review due to limitations of the available studies and potential limitation of the applicability of the data. The included studies are heterogeneous and relatively small; the variable proportions of patient sex, stress modality, and exact protocols preclude the performance of a formal metaanalysis. Notably, direct comparison of both tracers within the same patient is limited to a significant minority of very small studies. In the modern era of solid-state detectors using upright, semi-upright, and prone positioning, it is unclear how variable post-injection delays affect image quality. The impact of time from radiotracer injection to imaging is also unclear when attenuation correction is used with a conventional Anger camera system. Additionally, stress modality has a robust effect on the intensity of subdiaphragmatic interfering activity, with vasodilator pharmacologic stress agents producing more intense hepatobiliary activity than exercise. It is conceivable that if controlled for the variable of stress modality, the data may favor longer post-injection delays for both tracers under vasodilatory stress in order to optimize hepatic clearance.

A reality of modern healthcare delivery is that variables such as efficiency and patient satisfaction must be weighed against expense and cost-effective practice. Sestamibi, though best imaged with a longer delay, is currently available as a generic kit and may be more affordable than tetrofosmin which is brand name only at the time of this writing. According to one study included in the review with 686 patients, a shorter post-injection delay with tetrofosmin translated into a total single day rest-stress protocol that was on average only 34 minutes shorter. ${ }^{9}$ The full duration of a single day rest-stress SPECT MPI can be many hours, particularly with use of conventional cameras. Though a half an hour shorter test time would certainly be preferred by most patients, it is unclear if this degree of improved efficiency alone would outweigh a significant cost difference between the two tracers.

In summary, the two available Tc-99m labeled SPECT MPI tracers currently in use produce images of excellent diagnostic quality at reasonably low total radiation dose to patients. Duvall et al. provide an excellent systematic review supporting the hypothesis that more rapid blood pool and hepatic tracer clearance with tetrofosmin may allow for shorter post-injection delays to image acquisition and result in images of comparable diagnostic quality to later sestamibi imaging. A longer delay with tetrofosmin may, however, still be superior. The decision by nuclear cardiology laboratories of which SPECT MPI tracer to use should include considerations such as tracer price, lab efficiency, stress modality frequencies, and, possibly, type of imaging system used. Nuclear cardiology laboratories utilizing sestamibi may potentially benefit from a more competitive generic radiotracer cost, but this must be balanced against a possible improvement in laboratory efficiency and patient satisfaction with tetrofosmin. Laboratories already using tetrofosmin should consider the data on image quality carefully before considering shorter protocols. Beyond the scope of the present study, while image quality and patient convenience are important, the essential goals of myocardial perfusion imaging remain the accurate diagnosis of coronary artery disease and reliable assessment of prognosis. Further controlled study of how protocols with variable post-injection delays ultimately translate into diagnostic accuracy using invasive coronary angiography and predict patient outcomes would be a beneficial addition to the literature in response to this systematic review.

\section{Disclosure}

The authors receive grant funding from GE Healthcare for the unrelated GE AURORA study.

\section{References}

1. Beller GA, Watson DD. Physiological basis of myocardial perfusion imaging with the technetium99m agents. Semin Nucl Med 1991;12:173-81.

2. Sogbein OO, Pelletier-Galarneau M, Schindler TH, Wei L, Wells RG, Ruddy TD. New SPECT and PET radiopharmaceuticals for imaging cardiovascular disease. Biomed Res Int 2014. https://doi. org/10.1155/2014/942960.

3. Wackers FJ, Berman DS, Maddahi J, Watson DD, Beller GA, Strauss HW, et al. Technetium- ${ }^{99 \mathrm{~m}}$ hexakis 2-methoxyisobutyl isonitrile: Human biodistribution, dosimetry, safety, and preliminary comparison to thallium-201 for myocardial perfusion imaging. J Nucl Med 1989;30:301-11.

4. Munch G, Neverve J, Matsunari I, Schroter G, Schwaiger M. Myocardial technetium- ${ }^{99 \mathrm{~m}}$-tetrofosmin and technetium $-{ }^{-9 \mathrm{~m}}$-sestamibi kinetics in normal subjects and patients with coronary artery disease. J Nucl Med 1997;38:428-32.

5. Dorbala S, Ananthasubramaniam K, Armstrong IS, Chareonthaitawee P, DePuey EG, Einstein AJ, et al. Single photon emission computed tomography (SPECT) Myocardial Perfusion Imaging Guidelines: Instrumentation, acquisition, processing, and interpretation. J Nucl Cardiol 2018;25:1784-846.

6. Vitola JV, Shaw LJ, Allam AH, Orellana P, Peix A, Ellmann A, et al. Assessing the need for nuclear cardiology and other advanced cardiac imaging modalities in the developing world. J Nucl Cardiol 2009; 16:956-61.

7. Delbeke D, Segall GM. Status of and trends in nuclear medicine in the United States. J Nucl Med 2011;52:24S-8S.

8. Duvall WL, Case J, Lundbye J, Cerqueira M. Efficiency of tetrofosmin versus sestamibi achieved through shorter injection-to- 
imaging times: A systematic review of the literature. J Nucl Cardiol 2020. https://doi.org/10.1007/s12350-020-02093-5.

9. Ravizzini GC, Hanson MW, Shaw LK, Wong TZ, Hagge RJ, Pagnanelli RA, et al. Efficiency comparison between $99 \mathrm{~m} \mathrm{Tc}$ tetrofosmin and $99 \mathrm{~m}$ Tc-sestamibi myocardial perfusion studies.
Nucl Med Commun 2002;23:203-8. https://doi.org/10.1097/00006 231-200203000-00002.

Publisher's Note Springer Nature remains neutral with regard to jurisdictional claims in published maps and institutional affiliations. 